\title{
GENERALIZED BOUSFIELD LATTICES AND A GENERALIZED RETRACT CONJECTURE
}

\author{
RYO KATO, KATSUMI SHIMOMURA, AND YUTARO TATEHARA
}

\begin{abstract}
In [1], Bousfield studied a lattice (Bousfield lattice) on the stable homotopy category of spectra, and in [5], Hovey and Palmieri made the retract conjecture on the lattice. In this paper we generalize the Bousfield lattice and the retract conjecture to the ones on a monoid. We also determine the structure of typical examples of them, which satisfy the generalized retract conjecture. In particular we give the structure of the Bousfield lattice of the stable homotopy category of harmonic spectra explicitly.
\end{abstract}

\section{INTRODUCTION}

Let $\mathcal{M}$ be a closed symmetric monoidal category with zero object, and consider an object $M$ of it. We call the full subcategory $\langle M\rangle$ of $\mathcal{M}$ the Bousfield class of $M$ if it consists of objects $A$ of $\mathcal{M}$ such that $M A=0$ by its monoidal structure. Then we have a partial order on Bousfield classes by $\langle M\rangle \leq\langle N\rangle$ if every object of $\langle N\rangle$ is an object of $\langle M\rangle$. Then the subcategories $\langle S\rangle$ and $\langle O\rangle$ of the unit $S$ and the zero $O$ are the greatest and the least ones in the order, respectively. We call a collection of Bousfield classes a Bousfield lattice, and denote it by $\mathbb{B}(\mathcal{M})$. In a case where a Bousfield lattice is a set, the partial order introduces a lattice structure to it, and we may investigate it algebraically.

In a sense, the stable homotopy theory is analyzing stable homotopy categories (cf. [6]). A stable homotopy category is a symmetric monoidal category, and so we may consider its Bousfield lattice. In particular, T. Ohkawa [8] (cf. [2]) showed that the Bousfield lattice $\mathbb{B}$ of the stable homotopy category of spectra is a set, and then Iyengar and Krause [7] generalized it to a stable homotopy category.

In order to investigate a category, we sometimes classify special subcategories of it. From this viewpoint, we study a Bousfield lattice by classifying localizing subcategories (see [6]). Indeed, every Bousfield class is a localizing subcategory.

In [5], Hovey and Palmieri studied the Bousfield lattice $\mathbb{B}$ deeply. Furthermore, they proposed many conjectures on the structure of $\mathbb{B}$. Among them, there is the retract conjecture, which is one of our main topics. Dwyer and Palmieri [3] constructed a stable homotopy category, where the conjecture does not hold. So far, there seems no nontrivial category in which the conjecture holds. In this paper, we give some examples of categories with the affirmative answer to the conjecture.

As stated above, a Bousfield lattice $\mathbb{B}(\mathcal{M})$ is a set in some cases. In this case, it is a monoid with multiplication compatible with its order. We introduce the notion of monoidal posets and define a functor $\beta$ from a subcategory of commutative monoids to the category of monoidal posets in Section two. Then we define a Bousfield lattice of a monoid to be an object in the image of $\beta$, which is an analogy of Bousfield

2000 Mathematics Subject Classification. Primary 55P42, Secondary 06D75. 
lattices of stable homotopy categories. In particular, $\mathbb{B}$ has not only a structure of a monoidal poset, but also a Bousfield lattice associated to $\mathbb{B}$ itself. In section three, we show analogous properties on a Bousfield lattice to those given by Hovey and Palmieri [5] including the following:

Conjecture 1.1 (Original retract conjecture [5, Conj. 3.12]). Let h be the Bousfield class of the $\bmod p$ Eilenberg-MacLane spectrum $H \mathbb{Z} / p$ in the Bousfield lattice $\mathbb{B}$. Then, there is a lattice isomorphism $r_{*}: \mathbb{B} / J(h) \rightarrow D L$. Here, $J(h)$ is an ideal related to $h$ (see Notation 3.1).

We generalize it to generalized retract conjectures on a monoidally distributive poset (Conjectures 3.18 and 3.20) and show some facts relating to them. Section four is devoted to determine Bousfield lattices obtained from principal ideal domains, and to show the conjecture true for them. In section five, we study about Bousfield lattices of stable homotopy categories of Bousfield localized spectra, and construct isomorphisms between the Bousfield lattice and a Bousfield lattice given in section four. In particular, we have the following:

Theorem 1.2. The generalized retract conjectures holds on the stable homotopy category of harmonic spectra.

One of our final goals is to determine the lattice structure of $\mathbb{B}$, which seems difficult so much. In the last section, we propose problems on the functor $\beta$, whose answers may help us to understand the Bousfield lattice $\mathbb{B}$. We expect that these problems give us hints to reach the goal.

\section{Monoidal posets And Bousfield LATtices}

Let $M$ be commutative monoid with unit 1 . We call $M$ a monoid with 0 if $M$ admits an element $0 \in M$ such that $0 \cdot x=0=x \cdot 0$ for any $x \in M$. A typical example of it is a commutative ring ignoring addition. We denote by $\mathcal{M}_{0}$ the category consisting of commutative monoids with 0 and monoid homomorphisms preserving zero.

For $M \in \mathcal{M}_{0}, \beta(M)$ denotes a set consisting of subsets

$$
\langle x\rangle=\{y \in M: x y=0\}
$$

of $M$ for $x \in M$.

Lemma 2.1. $\beta(M)$ for $M \in \mathcal{M}_{0}$ is also a monoid with 0 with inherited multiplication. Therefore, we have the canonical epimorphism $M \rightarrow \beta(M)$ in $\mathcal{M}_{0}$.

Proof. Define a multiplication of $\beta(M)$ by $\langle x\rangle\langle y\rangle=\langle x y\rangle$. We verify it well defined as follows: Assume that $\left\langle x_{0}\right\rangle=\left\langle x_{1}\right\rangle$ and $\left\langle y_{0}\right\rangle=\left\langle y_{1}\right\rangle$. Then

$$
\begin{aligned}
z x_{0} y_{0}=0 & \Leftrightarrow z x_{1} y_{0}=0 \quad \text { by }\left\langle x_{0}\right\rangle=\left\langle x_{1}\right\rangle \\
& \Leftrightarrow z x_{1} y_{1}=0 \quad \text { by }\left\langle y_{0}\right\rangle=\left\langle y_{1}\right\rangle,
\end{aligned}
$$

and $\left\langle x_{0} y_{0}\right\rangle=\left\langle x_{1} y_{1}\right\rangle$. The elements $\langle 1\rangle$ and $\langle 0\rangle$ are the unit and the zero elements.

Remark 2.2. We notice that $\beta(R)=\mathbb{Z} / 2$ if $R$ is a domain.

Lemma 2.3. Let $M$ be a monoid with 0 . Then $\beta(M)$ admits a partial order ' $\leq$ ' on $M$ defined by $\langle x\rangle \leq\langle y\rangle$ if $\langle x\rangle \supset\langle y\rangle$. Besides, $\langle 1\rangle$ and $\langle 0\rangle$ are the greatest and the least elements, respectively. 
Proof. This is trivial since $\langle 1\rangle=\{0\}$ and $\langle 0\rangle=M$.

By the lemma, a commutative monoid $\beta(M)$ has also a poset structure. Then we define the following notion by taking their crucial properties.

Definition 2.4. A monoidal poset $P=(P, \leq, \cdot, 1,0)$ is defined by the following data.

(1) $(P, \cdot, 1,0)$ is a monoid with 0 .

(2) $(P, \leq)$ is a poset.

(3) The following are equivalent.

(a) $x \leq y$.

(b) $c y=0$ for $c \in P$ implies $c x=0$.

A monoidal poset map $f: P \rightarrow P^{\prime}$ is an order preserving monoid homomorphism with $f(0)=0$.

Lemma 2.3 implies the following.

Corollary 2.5. $\beta(M)$ for $M \in \mathcal{M}_{0}$ is a monoidal poset with $1=\langle 1\rangle$ and $0=\langle 0\rangle$.

Lemma 2.6. Let $M$ be a monoidal poset. Then, $\beta(M)=M$ as a monoidal poset.

Remark 2.7. A monoidal poset seems a lattice, but unfortunately it is not true. Indeed, we have an example: Consider a monoidal poset $M=\left\{1, x_{i}, y_{i}, w, 0: i=\right.$ $1,2\}$ with multiplication

\begin{tabular}{|c|c|c|c|c|c|}
\hline 1 & $x_{1}$ & $x_{2}$ & $y_{1}$ & $y_{2}$ & $w$ \\
\hline$x_{1}$ & $w$ & $w$ & 0 & $w$ & 0 \\
\hline$x_{2}$ & $w$ & $w$ & $w$ & 0 & 0 \\
\hline$y_{1}$ & 0 & $w$ & 0 & 0 & 0 \\
\hline$y_{2}$ & $w$ & 0 & 0 & 0 & 0 \\
\hline$w$ & 0 & 0 & 0 & 0 & 0 \\
\hline
\end{tabular}

Then, the join of $y_{1}$ and $y_{2}$ does not exist.

Let $\mathcal{M P}$ denote the category of monoidal posets and monoidal poset maps. Then $\mathcal{M P} \subset \mathcal{M}_{0}$.

Lemma 2.8. Let $M$ be a monoidal poset. Then, $x z \leq y w$ if $x \leq y$ and $z \leq w$. In particular, if $x \leq y$, then $x z \leq y z$ for any $z$.

Proposition 2.9. The category $\mathcal{M P}$ admits a direct product.

Proof. Let $\left\{M_{\lambda}\right\}$ be a family of monoidal posets. Then, we have a direct product $\prod_{\lambda} M_{\lambda}$ of monoids. Consider an order ' $\leq$ ' on $\prod_{\lambda} M_{\lambda}$ defined by $\left(x_{\lambda}\right) \leq\left(y_{\lambda}\right)$ if $\left(c_{\lambda}\right)\left(y_{\lambda}\right)=(0)$ implies $\left(c_{\lambda}\right)\left(x_{\lambda}\right)=(0)$. It is straightforward to verify this is the desired direct product.

Lemma 2.10. Let $\left\{M_{\lambda}\right\}$ be a family of monoidal posets. Then, $\left\langle x_{\lambda}\right\rangle \leq\left\langle y_{\lambda}\right\rangle$ for all $\lambda$ if and only if $\left\langle\left(x_{\lambda}\right)\right\rangle \leq\left\langle\left(y_{\lambda}\right)\right\rangle$. Here, $\left\langle x_{\lambda}\right\rangle,\left\langle y_{\lambda}\right\rangle \in \beta\left(M_{\lambda}\right)$ and $\left\langle\left(x_{\lambda}\right)\right\rangle,\left\langle\left(y_{\lambda}\right)\right\rangle \in$ $\beta\left(\prod_{\lambda} M_{\lambda}\right)$.

Proof. Assume that $\left\langle x_{\lambda}\right\rangle \leq\left\langle y_{\lambda}\right\rangle$ for any $\lambda$. Then

$$
\begin{aligned}
\left(c_{\lambda}\right)\left(y_{\lambda}\right)=0 & \Rightarrow c_{\lambda} y_{\lambda}=0 \text { for any } \lambda \\
& \Rightarrow c_{\lambda} x_{\lambda}=0 \text { for any } \lambda\left(\because\left\langle x_{\lambda}\right\rangle \leq\left\langle y_{\lambda}\right\rangle\right) \\
& \Rightarrow\left(c_{\lambda}\right)\left(x_{\lambda}\right)=0,
\end{aligned}
$$


Conversely, suppose that $\left\langle\left(x_{\mu}\right)\right\rangle \leq\left\langle\left(y_{\mu}\right)\right\rangle$. Then, for any $\lambda$,

$$
\begin{aligned}
y_{\lambda} c_{\lambda}=0 & \Rightarrow\left(y_{\lambda}\right)\left(c_{\lambda}\right)_{0}=0 \\
& \Rightarrow\left(x_{\lambda}\right)\left(c_{\lambda}\right)_{0}=0\left(\because\left\langle\left(x_{\mu}\right)\right\rangle \leq\left\langle\left(y_{\mu}\right)\right\rangle\right) \\
& \Rightarrow x_{\lambda} c_{\lambda}=0
\end{aligned}
$$

in $M_{\lambda}$, where $\left(c_{\lambda}\right)_{0}$ denotes an element $\left(x_{\mu}\right)$ such that $x_{\lambda}=c_{\lambda}$ and $x_{\mu}=0$ for $\mu \neq \lambda$.

Corollary 2.11. Let $\left\{M_{\lambda}\right\}$ be a family of monoidal posets. Define an order $\leq^{\prime}$ on the set $\prod_{\lambda} M_{\lambda}$ by $\left(x_{\lambda}\right) \leq^{\prime}\left(y_{\lambda}\right)$ if $x_{\lambda} \leq y_{\lambda}$ for all $\lambda$. Then it is equivalent to the order above $\leq$.

Corollary 2.12. Let $\left\{M_{\lambda}\right\}$ be a family of monoidal posets. Then, $\bigvee_{\mu}\left(x_{\lambda}^{\mu}\right)=$ $\left(\bigvee_{\mu} x_{\lambda}^{\mu}\right)$ for any subset $\left\{\left(x_{\lambda}^{\mu}\right)\right\}_{\mu} \subset \prod_{\lambda} M_{\lambda}$.

Proof. Since $\left(x_{\lambda}^{\mu}\right) \leq\left(\bigvee_{\mu} x_{\lambda}^{\mu}\right)$ for all $\mu, \bigvee_{\mu}\left(x_{\lambda}^{\mu}\right) \leq\left(\bigvee_{\mu} x_{\lambda}^{\mu}\right)$. If $\left(x_{\lambda}^{\mu}\right) \leq\left(z_{\lambda}\right)$, then $x_{\lambda}^{\mu} \leq z_{\lambda}$, and so $\bigvee_{\mu} x_{\lambda}^{\mu} \leq z_{\lambda}$, that is, $\left(\bigvee_{\mu} x_{\lambda}^{\mu}\right) \leq\left(z_{\lambda}\right)$. Therefore, $\bigvee_{\mu}\left(x_{\lambda}^{\mu}\right)=\left(\bigvee_{\mu} x_{\lambda}^{\mu}\right)$ by definition.

We call an epimorphism $f: M \rightarrow N$ of $\mathcal{M}_{0}$ strong if $f(x)=0$ if and only if $x=0$.

We define a map $\beta(f): \beta(M) \rightarrow \beta(N)$ by assigning $\langle x\rangle$ to $\langle f(x)\rangle$.

Lemma 2.13. For a strong epimorphism $f: M \rightarrow N$, the map $\beta(f)$ is not only a monoidal poset map but also a strong epimorphism.

Proof. Since $f$ is a strong epimorphism, $c \cdot f(x)=0 \Leftrightarrow f\left(c^{\prime}\right) \cdot f(x)=0 \Leftrightarrow f\left(c^{\prime} \cdot x\right)=$ $0 \Leftrightarrow c^{\prime} \cdot x=0$ for an element $c^{\prime}$ such that $f\left(c^{\prime}\right)=c$. This shows that $\langle x\rangle=\langle y\rangle$ implies $\langle f(x)\rangle=\langle f(y)\rangle$. It is easy to see that $\beta(f)$ is a strong epimorphism.

We also consider the subcategories $\mathcal{M}_{0}^{\text {epi }}$ and $\mathcal{M} \mathcal{P}^{\text {epi }}$ of $\mathcal{M}_{0}$ and $\mathcal{M P}$, respectively, obtained by restricting morphisms to strong epimorphisms.

Corollary 2.14. The operation $\beta$ above defines a functor $\beta: \mathcal{M}_{0}^{\text {epi }} \rightarrow \mathcal{M P}^{\text {epi }} \subset$ $\mathcal{M}_{0}^{e p i}$.

By the above argument, we redefine Bousfield lattices as follows. The definition is one of our main topics in this paper.

Definition 2.15. For a monoid $M \in \mathcal{M}_{0}^{e p i}$ we call a monoidal poset $\beta(M)$ the Bousfield lattice associated to $M$.

In earlier papers, a Bousfield lattice is made from a closed symmetric monoidal category with a zero object. However, their set theoretic confusion complicates our argument too much. Our new definition settles this problem, and the following proposition says that this argument is consistent.

Proposition 2.16. The Bousfield lattice $\mathbb{B}$ of the stable homotopy category of spectra is a Bousfield lattice in the sense of our definition.

Proof. By forgetting the ordering on $\mathbb{B}$, we regard $\mathbb{B}$ as a monoid with $1=\langle S\rangle$ and $0=\langle *\rangle$. Then it is clear that $\beta(\mathbb{B})=\mathbb{B}$.

Proposition 2.17. The functor $\beta$ satisfies the following: 
(1) $\beta\left(\prod_{\lambda} M_{\lambda}\right)=\prod_{\lambda} \beta\left(M_{\lambda}\right)$.

(2) $\beta \beta(M)=\beta(M)$.

Proof. (1) Let $\left\{p_{\lambda}: \beta\left(\prod_{\lambda} M_{\lambda}\right) \rightarrow \beta\left(M_{\lambda}\right)\right\}$ be a family of epimorphisms defined by $\left\langle\left(x_{\lambda}\right)\right\rangle \mapsto\left\langle x_{\lambda}\right\rangle$, and $\left\{f_{\lambda}: W \rightarrow \beta\left(M_{\lambda}\right)\right\}$ a family of poset maps. We notice that $p_{\lambda}$ is well defined by Lemma 2.10. For an element $w \in W$, we take an element $w_{\lambda} \in W_{\lambda}$ so that $f_{\lambda}(w)=\left\langle w_{\lambda}\right\rangle$, and define $g: W \rightarrow \beta\left(\prod_{\lambda} M_{\lambda}\right)$ by $g(w)=\left\langle\left(w_{\lambda}\right)\right\rangle$. Then $g$ is also a well defined poset map by Lemma 2.10 and

$$
p_{\lambda} g(w)=p_{\lambda}\left(\left\langle\left(w_{\lambda}\right)\right\rangle\right)=\left\langle w_{\lambda}\right\rangle=f_{\lambda}(w) .
$$

Suppose that there is another poset map $g^{\prime}: W \rightarrow \beta\left(\prod_{\lambda} M_{\lambda}\right)$ satisfying $p_{\lambda} g^{\prime}(w)=$ $f_{\lambda}(w)$ for $w \in W$, and $g^{\prime}$ assigns $w$ to $\left\langle\left(w_{\lambda}^{\prime}\right)\right\rangle$. Then

$$
\begin{aligned}
p_{\lambda} g^{\prime}(w)=f_{\lambda}(w) \text { for any } \lambda & \Leftrightarrow\left\langle w_{\lambda}^{\prime}\right\rangle=\left\langle w_{\lambda}\right\rangle \text { for any } \lambda \\
& \Leftrightarrow\left\langle\left(w_{\lambda}^{\prime}\right)\right\rangle=\left\langle\left(w_{\lambda}\right)\right\rangle(\because \text { Lemma } 2.10) \\
& \Leftrightarrow g^{\prime}(w)=g(w) .
\end{aligned}
$$

Therefore, $\beta\left(\prod_{\lambda} M_{\lambda}\right)$ is the product $\prod_{\lambda} \beta\left(M_{\lambda}\right)$.

(2) is seen by Lemma 2.6 .

\section{Retract conjecture}

From now on, we assume that every monoidal poset is a complete lattice.

Since a monoidal poset $M$ is a sup-lattice with the least element $0=\langle 0\rangle, M$ is a bounded lattice.

Notation 3.1. For a monoidal poset $M$, we define the following notations.

$$
\begin{aligned}
a_{M}(x) & :=\bigvee\{y \in M: x y=0\} \text { for } x \in M, \\
D L(M) & :=\left\{x \in M: x^{2}=x\right\}, \\
r_{M}(x) & :=\bigvee\{w \in D L(M): w \leq x\} \text { for } x \in M, \\
J_{M}(x) & :=\left\{y \in M: y \leq x \wedge a_{M}(x)\right\} \text { for } x \in M, \\
N(M) & :=\left\{x \in M: x^{n}=0 \text { for some } n \geq 1\right\}, \\
A(M) & :=\left\{x \in M: r_{M}(x)=0\right\} .
\end{aligned}
$$

We will omit $M$ from notations, if $M$ is clear from the context.

The subposet $D L(M)$ is also a complete lattice. Indeed the following holds.

Proposition 3.2. $D L(M)$ is closed under the join $\vee$.

Proof. By Lemma 2.8, $\left(\bigvee_{\lambda \in \Lambda} x_{\lambda}\right)^{2} \leq\left(\bigvee_{\lambda \in \Lambda} x_{\lambda}\right)$. Suppose that $x_{\lambda}$ is in $D L$ for $\lambda \in \Lambda$. Then, $x_{\lambda}=x_{\lambda}^{2} \leq\left(\bigvee_{\lambda \in \Lambda} x_{\lambda}\right)^{2}$, and so $\bigvee_{\lambda \in \Lambda} x_{\lambda} \leq\left(\bigvee_{\lambda \in \Lambda} x_{\lambda}\right)^{2}$.

Lemma 3.3. In $D L(M)$, the meet of $x$ and $y$ is $x y$.

Proof. Since $x \wedge y \leq x$ and $x \wedge y \leq y$, if $x \wedge y \in D L(M)$ then $x \wedge y \leq x y$.

Remark 3.4. $D L(M)$ is not always sublattice of $M$ by Lemma 3.3 .

For investigating the original Bousfield lattice $\mathbb{B}$, the operations $r$ and $a$ play important roles (see [5]). Hereafter we try to give their properties analogously on good monoidal posets.

Proposition 3.5. Let $M$ be a monoidal poset, and $r=r_{M}: M \rightarrow M$ be the map defined in Notation 3.1.

(1) $r$ is order-preserving i.e. $x \leq y$ implies $r(x) \leq r(y)$. 
(2) $r(x)^{2}=r(x)$ and $r^{2}(x)=r(x)$ for $x \in M$.

(3) $r(x) \leq x^{n}$ for any $n \geq 1$.

(4) $r(x y)=r(x) r(y)=r(x \wedge y)$ for $x, y \in M$.

Proof. (1) is trivial, and (2) follows from Proposition 3.2. For (3), $r(x) \leq x$ by definition, and we have $r(x)=r(x)^{n} \leq x^{n}$.

Since $r(x) r(y) \leq x y$ and $r(x) r(y) \in D L(M)$, we have $r(x) r(y) \leq r(x y)$. We also see $r(x \wedge y) \leq r(x) r(y)$, since $r(x \wedge y) \leq r(x)$ and $r(x \wedge y) \leq r(y)$. Therefore, $r(x y) \leq r(x \wedge y) \leq r(x) r(y) \leq r(x y)$, and obtain (4).

The behavior of the map $r$ is the same as the one on $\mathbb{B}$, but not that of the operation $a$. Indeed, for any $x \in M$ and $\left\{y_{\lambda}\right\}_{\lambda} \subset M$, the relation $x\left(\bigvee_{\lambda} y_{\lambda}\right) \geq$ $\bigvee_{\lambda}\left(x y_{\lambda}\right)$ is not always equal. To make the operator $a$ have a good nature, we introduce a following notion.

Definition 3.6. A monoidal poset $M$ is a monoidally distributive poset if $M$ satisfies that $x\left(\bigvee_{\lambda} y_{\lambda}\right)=\bigvee_{\lambda}\left(x y_{\lambda}\right)$ for any $x \in M$ and $\left\{y_{\lambda}\right\}_{\lambda} \subset M$.

Remark 3.7. $D L(M)$ is a distributive lattice if $M$ is a monoidally distributive poset by Lemma 3.3 .

In the same way as [5], we have

Proposition 3.8. Let $M$ be a monoidally distributive poset. Then,

(1) $a(-)$ is order-reversing.

(2) $x y=0$ if and only if $x \leq a(y)$.

(3) $a a(x)=x$.

Lemma 3.9. Let $M$ be a monoidally distributive poset. Fix $c \in M$ such that $c^{n}=0$ for a positive integer $n$. Then, for any $x \in M,(x \vee c)^{n} \leq x$ and $r(x \vee c)=r(x)$.

Proof. Under the assumption, we compute

$$
\begin{aligned}
(x \vee c)^{n} & =x^{n} \vee x^{n-1} c \vee \cdots \vee x c^{n-1} \\
& =x\left(x^{n-1} \vee x^{n-2} c \vee \cdots \vee c^{n-1}\right) \leq x
\end{aligned}
$$

for any $x \in M$. So, if $z \leq x \vee c$ for $z \in D L(M)$, then $z \leq x$. Thus, $r(x \vee c)=r(x)$ by definition of $r$.

Proposition 3.10. Let $M$ be a monoidally distributive poset. Then $J_{M}(x) \subset$ $N(M) \subset A(M)$ for any $x \in M$.

Proof. Since $\left(x \wedge a_{M}(x)\right)\left(x \wedge a_{M}(x)\right) \leq x a_{M}(x)=0$ by Proposition 3.8(2), we have $J_{M}(x) \subset N(M)$. Suppose that $x^{n}=0$, then $r(x)=r(x)^{n}=r\left(x^{n}\right)=r(0)=0$ by Proposition 3.5 (4). So we have $N(M) \subset A(M)$.

Proposition 3.11. Let $M_{\lambda}$ be a monoidal poset for any $\lambda \in \Lambda$. Then,

(1) $r\left(\left(x_{\lambda}\right)\right)=\left(r\left(x_{\lambda}\right)\right)$ for any $\left(x_{\lambda}\right) \in \prod_{\lambda} M_{\lambda}$.

(2) $r$ preserves arbitrary joins on $M_{\lambda}$ for any $\lambda \in \Lambda$ if and only if $r$ preserves arbitrary joins on $\prod_{\lambda} M_{\lambda}$

Proof. (1) is given by corollary 2.12 . 
(2) Suppose that $r$ preserves arbitrary joins on $M_{\lambda}$ for any $\lambda \in \Lambda$. Then, for $\left\{\left(x_{\lambda}^{\mu}\right)\right\}_{\mu} \subset \prod_{\lambda} M_{\lambda}$

$$
\begin{aligned}
r\left(\bigvee_{\mu}\left(x_{\lambda}^{\mu}\right)\right) & =r\left(\left(\bigvee_{\mu} x_{\lambda}^{\mu}\right)\right)(\because \text { corollary 2.12) } \\
& =\left(r\left(\bigvee_{\mu} x_{\lambda}^{\mu}\right)\right)(\because(1)) \\
& =\left(\bigvee_{\mu} r\left(x_{\lambda}^{\mu}\right)\right) \\
& =\bigvee_{\mu}\left(r\left(x_{\lambda}^{\mu}\right)\right) \quad(\because \text { corollary 2.12) }
\end{aligned}
$$

Therefore, $r$ preserves arbitrary joins on $\prod_{\lambda} M_{\lambda}$.

Conversely, under the assumption,

$$
\begin{aligned}
\left(r\left(\bigvee_{\mu} x_{\lambda}^{\mu}\right)\right) & =r\left(\left(\bigvee_{\mu} x_{\lambda}^{\mu}\right)\right) \quad(\because(1)) \\
& =r\left(\bigvee_{\mu}\left(x_{\lambda}^{\mu}\right)\right) \quad(\because \text { corollary 2.12) } \\
& =\bigvee_{\mu}\left(r\left(x_{\lambda}^{\mu}\right)\right) \\
& =\left(\bigvee_{\mu} r\left(x_{\lambda}^{\mu}\right)\right) \quad(\because \text { corollary 2.12) }
\end{aligned}
$$

It follows that $r$ preserves arbitrary joins on $M_{\lambda}$ for any $\lambda \in \Lambda$ as desired.

Remark 3.12. We notice that $M_{\lambda}$ is a monoidally distributive poset for any $\lambda \in \Lambda$ if and only if $\prod_{\lambda \in \Lambda} M_{\lambda}$ is a monoidally distributive poset. Indeed, if $M_{\lambda}$ is a monoidally distributive poset for any $\lambda \in \Lambda$, then $\left(c_{\lambda}\right)\left(\bigvee_{\mu}\left(x_{\lambda}^{\mu}\right)\right)=\left(c_{\lambda}\right)\left(\bigvee_{\mu} x_{\lambda}^{\mu}\right)=$ $\left(c_{\lambda}\left(\bigvee_{\mu} x_{\lambda}^{\mu}\right)\right)=\left(\bigvee_{\mu} c_{\lambda} x_{\lambda}^{\mu}\right)=\bigvee_{\mu}\left(c_{\lambda} x_{\lambda}^{\mu}\right)$ for $\left(c_{\lambda}\right) \in \prod_{\lambda} M_{\lambda}$ and $\left\{\left(x_{\lambda}^{\mu}\right)\right\}_{\mu} \subset \prod_{\lambda} M_{\lambda}$ by corollary 2.12. Thus, $\prod_{\lambda} M_{\lambda}$ is a monoidally distributive poset. Conversely, under the assumption $\left(c_{\lambda}\left(\bigvee_{\mu} x_{\lambda}^{\mu}\right)\right)=\left(c_{\lambda}\right)\left(\bigvee_{\mu} x_{\lambda}^{\mu}\right)=\left(c_{\lambda}\right)\left(\bigvee_{\mu}\left(x_{\lambda}^{\mu}\right)\right)=\bigvee_{\mu}\left(c_{\lambda} x_{\lambda}^{\mu}\right)=$ $\left(\bigvee_{\mu} c_{\lambda} x_{\lambda}^{\mu}\right)$ by corollary 2.12. Therefore, $M_{\lambda}$ is a monoidally distributive poset for any $\lambda \in \Lambda$ by Lemma 2.10 .

Recall that an ideal $I$ of a poset is defined by

(1) If $x \in I$, and $y \leq x$, then $y \in I$, and

(2) For $x, y \in I$, there is an element $z \in I$ such that $x \leq z$ and $y \leq z$.

Suppose that a monoidal poset $M$ is an ordinary lattice. Then, an ideal of $M$ is also an ideal as a lattice, and for an ideal $I, M / I$ is the lattice of equivalent classes under the equivalent relation defined by

$$
x \sim y \quad \text { if and only if } x \vee c=y \vee c \text { for some } c \in I
$$

with order given by $[x] \leq[y] \Leftrightarrow x \vee c \leq y \vee c$ for some $c \in I$. We notice that $M / I$ is complete if $M$ and $I$ are complete. If $M$ is monoidally distributive, then $M / I$ has the multiplication $[x][y]:=[x y]$. Indeed, if $x \vee i=x^{\prime} \vee i$ and $y \vee j=y^{\prime} \vee j$ for $x, x^{\prime}, y, y^{\prime} \in M$ and $i, j \in I$, then $(x \vee i)(y \vee j)=\left(x^{\prime} \vee i\right)\left(y^{\prime} \vee j\right)$ turns into

$$
\begin{aligned}
x y \vee(x \vee i) j \vee(y \vee j) i & =x^{\prime} y^{\prime} \vee\left(x^{\prime} \vee i\right) j \vee\left(y^{\prime} \vee j\right) i \\
& =x^{\prime} y^{\prime} \vee(x \vee i) j \vee(y \vee j) i .
\end{aligned}
$$

Since $(x \vee i) j \vee(y \vee j) i \in I$, the multiplication is well defined.

Remark 3.14. $M / I$ is not always a monoidal poset. Indeed, we have an example: Let $M=\{1, x, y, 0\}$ be a monoidal poset with multiplication $x^{2}=x, x y=0, y^{2}=0$. Then, for the ideal $I=\{y, 0\}, M / I=\{1, x, 0\}$ and $\beta(M / I)=\{1,0\}$. Since $M / I \neq \beta(M / I), M / I$ is not a monoidal poset by Lemma 2.6 .

Lemma 3.15. Let $M$ be a monoidally distributive poset. Then, $N(M)$ is an ideal of $M$ and $J_{M}(x)$ is a principal ideal of $M$ for any $x \in M$. 
Proof. Suppose that $x^{n}=0$ and $y^{m}=0$. Then, $(x \vee y)^{n+m}=\bigvee_{a+b=n+m} x^{a} y^{b}$. Since if $a<n$ then $b \geq m,(x \vee y)^{n+m}=0$. So $N(M)$ is an ideal of $M$. By definition, $J_{M}(x)$ is a principal ideal of $M$.

Here, consider the following correspondence:

$$
r_{*}: M / I \rightarrow D L(M) ;[x] \mapsto\{r(y): y \in[x]\}
$$

We notice that $r_{*}$ is surjection if $r_{*}$ is a mapping.

Theorem 3.16. Let $M$ be a monoidally distributive poset and $I$ an ideal in $M$.

(1) If $I$ is contained in $N$, then $r_{*}$ is a mapping.

(2) If $r_{*}$ is a mapping, then $I \subset A$.

(3) If $r_{*}$ is an injection, then $I=A$.

(4) If $r_{*}$ is an injection and $I \subset N$, then:

(a) For any $x$ and $y$ in $M, r(x \vee y)=r(x) \vee r(y)$ holds. In particular, if $I$ is a principal ideal, then $r$ preserves arbitrary joins.

(b) For any $x \in M$, there exists an integer $n$ such that $x^{n}=r(x)$.

Proof. (1) If $x \vee c=y \vee c$ for $x, y \in M$ and $c \in I \subset N$, then $r(x)=r(y)$ by Lemma 3.9.

(2) For $x \in I,[x]=0=[0]$ in $M / I$, and so $r(x)=r_{*}([x])=r_{*}([0])=r(0)=0$. Thus, $x \in A$.

(3) For $x \in A, r_{*}([x])=r(x)=0=r_{*}([0])$. It follows that $[x]=[0]$, since $r_{*}$ is an injection, which implies $x \in I$. So we obtain $A=I$ by (2).

(4) For $x \in M, r_{*}([x])=r(x)=r^{2}(x)=r_{*}([r(x)])$ and $[x]=[r(x)]$, since $r_{*}$ is an injection. So we have an element $c_{x} \in N$ such that $x \vee c_{x}=r(x) \vee c_{x}$, and then:

(a) Since $x \vee y \vee c_{x} \vee c_{y}=r(x) \vee r(y) \vee c_{x} \vee c_{y}, r(x \vee y)=r(x) \vee r(y)$ by Lemma 3.9. Suppose that $I$ is a principal ideal and take a generator $m$ of $I$. Then, $\left(\bigvee_{\lambda} x_{\lambda}\right) \vee m=\left(\bigvee_{\lambda} r\left(x_{\lambda}\right)\right) \vee m$ for any subset $\left\{x_{\lambda}\right\}_{\lambda} \subset M$. Therefore $r\left(\bigvee_{\lambda \in \Lambda} x_{\lambda}\right)=\bigvee_{\lambda \in \Lambda} r\left(x_{\lambda}\right)$ by Lemma 3.9.

(b) Since there exists an integer $n$ such that $c_{x}^{n}=0$,

$$
x^{n} \leq\left(x \vee c_{x}\right)^{n}=\left(r(x) \vee c_{x}\right)^{n} \leq r(x) .
$$

by Lemma 3.9.

Hovey and Palmieri introduced a map $r_{*}: M / J(h) \rightarrow D L$, and proposed Conjecture 1.1 in the introduction. Here, we generalize the map to our setting.

Lemma 3.17. The map $r_{M}: M \rightarrow M$ for a monoidal poset $M$ factors through $D L(M)$. Furthermore, it induces the map $r_{*}: M / J_{M}(y) \rightarrow D L(M)$ for $y \in M$ assigning the class $[x]$ to $r_{M}(x)$.

Proof. The former statement follows from Proposition 3.5(2), and the latter from Proposition 3.10 and Proposition 3.16(1).

By Theorem 3.16, we see that $J(h)=A$ if Conjecture 1.1 holds. This makes us conjecture the following:

Conjecture 3.18 (Generalized retract conjecture 1 (GRC1)). Let $M$ be a monoidal poset. If $M$ is a complete lattice and monoidally distributive, then $r_{*}: M / A \rightarrow D L$ is a lattice isomorphism.

Remark 3.19. We notice that $A(M)$ is not always an ideal of a monoidal poset $M$. GRC1 is false unless $A(M)$ is an ideal. 
Conjecture 3.20 (Generalized retract conjecture 2 (GRC2)). Let $M$ be a monoidal poset. If $M$ is a complete lattice and monoidally distributive, then $r_{*}: M / N \rightarrow$ $D L(M)$ is a lattice isomorphism.

By Theorem 3.16 (3), we see the following:

Corollary 3.21. GRC2 implies GRC1.

Example 3.22. Consider a monoidal poset $M=\beta\left(\mathbb{Z} / 2^{m} \mathbb{Z}\right)$. Then,

$$
\begin{aligned}
M & =\left\{1,2,2^{2}, \cdots, 2^{m-1}, 2^{m}=0\right\} \\
D L(M) & =\{1,0\} \text { and } \\
N(M) & =\left\{2,2^{2}, \cdots, 2^{m-1}, 0\right\} .
\end{aligned}
$$

And so $M / N(M) \cong D L(M)$. That is, GRC2 holds on $\beta\left(\mathbb{Z} / 2^{m} \mathbb{Z}\right)$.

Theorem 3.23. For a monoidally distributive poset $M$, the following are equivalent.

(1) $r_{*}: M / N \rightarrow D L$ is an isomorphism.

(2) Any class $[x] \in M / N$ satisfies $\left[x^{2}\right]=[x]$.

Proof. The statement (1) implies (2), since $r_{*}([x])=r_{*}\left(\left[x^{2}\right]\right)$.

For the converse, it suffices to show that $r_{*}$ is injective. If $\left[x^{2}\right]=[x]$, then $[x]=\left[x^{n}\right]$ for any $n>0$ by induction. So, we have an element $c_{x} \in N$ for each $x \in M$ such that

$$
x \vee c_{x}=x^{n} \vee c_{x} \text { for any } n>0 .
$$

Since $c_{x} \in N$, we have an integer $L=L(x)>0$ such that $c_{x}^{L}=0$. Then

$$
x^{L} \leq\left(x \vee c_{x}\right)^{L}=\left(x^{n} \vee c_{x}\right)^{L} \leq x^{n}
$$

for any $n>0$ by Lemma 3.9. In particular, $x^{L}=\left(x^{L}\right)^{2}$ and so

$$
x^{L(x)}=r(x) .
$$

by Proposition 3.5.

Now suppose that $r_{*}([x])=r_{*}([y])$. Then $r(x)=r(y)$, and $x^{L(x)}=y^{L(y)}$ by (3.25). By (3.24),

$$
x \vee c_{x} \vee c_{y}=x^{L(x)} \vee c_{x} \vee c_{y}=y^{L(y)} \vee c_{y} \vee c_{x}=y \vee c_{x} \vee c_{y}
$$

and $[x]=[y]$ by the definition (3.13).

Furthermore, Proposition 3.11 leads us to the following.

Proposition 3.26. Let $\left\{M_{\lambda}\right\}_{\lambda \in \Lambda}$ be a family of monoidally distributive posets. Then, the following are equivalent.

(1) GRC holds on $M_{\lambda}$ for any $\lambda \in \Lambda$.

(2) GRC holds on $\prod M_{\lambda}$.

Here, GRC is GRC1 or GRC2.

As an application, we extend a result of Dwyer and Palmieri:

Theorem 3.27 (Dwyer-Palmieri [3]). There is a ring $\Lambda$ such that a derived category $D(\Lambda)$ on $\Lambda$ does not hold the retract conjecture. 
In the proof of it, Dwyer and Palmieri define $\Lambda$ to be a truncated polynomial ring over a field $k$, and take $\langle k\rangle$ instead of $h=\langle H \mathbb{Z} / p\rangle$. Here $\langle k\rangle$ denotes a Bousfield class of a complex $\left\{X_{i}\right\}$ with $X_{0}=k$, and $X_{i}=0$ if $i \neq 0$. By a similar argument of Hovey and Palmieri in [5], if $r_{*}$ is an isomorphism from $\mathbb{B}(D(\Lambda)) / J(\langle k\rangle)$ to $D L$, then any Bousfield class $x \in \mathbb{B}(D(\Lambda))$ satisfies $x^{2}=x^{3}$. They show the theorem by constructing a Bousfield class $y \in \mathbb{B}(D(\Lambda))$ such that $y>y^{2}>\cdots>y^{n}>\cdots$. By Theorem 3.16, the existence of the class $y$ implies further the following:

Theorem 3.28. The map $r_{*}: \mathbb{B}(D(\Lambda)) / N \rightarrow D L$ is not isomorphic.

\section{A Bousfield lattice associated to A Quotient of PID}

We abbreviate 'principal ideal domain' to 'PID'. Furthermore, we write $x$ for $\langle x\rangle \in \beta(M)$, where no confusion arises.

Theorem 4.1. Let $P$ be a PID and put $q=p_{0}^{e_{0}} \cdots p_{m-1}^{e_{m-1}} \in P$ for prime elements $p_{i}$ and integers $e_{i}>0$. Let $B$ denote a Bousfield lattice $\beta(P / q P)$. Then,

(1) $B=\{x \in P: x \mid q\}$ as a set. In particular $q$ is the zero element 0 .

(2) $x \geq y$ if and only if $x \mid y$.

(3) $D \bar{L}=\left\{p_{0}^{s_{0}} \cdots p_{m-1}^{s_{m-1}}: s_{i}=0\right.$ or $\left.e_{i}\right\}$.

(4) $N=\left\{x \in B: p_{0} \cdots p_{m-1} \mid x\right.$ in $\left.P\right\}$.

(5) $B=\prod_{i=0}^{n-1} \beta\left(P / p_{i}^{e_{i}} P\right)$.

Proof. For an element $x \in P$, we consider an integer $e_{i}(x)$ and an element $x_{(q)}$ defined by

$$
e_{i}(x):=\max \left\{e: e \leq e_{i} \text { and } p_{i}^{e} \mid x\right\}, \quad \text { and } \quad x_{(q)}:=\prod_{0 \leq i<m} p_{i}^{e_{i}(x)} .
$$

We see that

$$
x=x_{(q)} \in \beta(P / q P) \quad \text { for any } x \in P .
$$

Indeed, $x_{(q)}$ divides $x$, and so $x \leq x_{(q)}$. If $x y=0$ in $P / q P$, then $x y$ is divided by $q$ in $P$. Therefore, $q \mid x_{(q)} y_{(q)}$ and so $q \mid x_{(q)} y$. Hence $x_{(q)} y=0$ in $P / q P$ and so $x_{(q)} \leq x$.

The statements (1)-(4) follow immediately from (4.2), and (5) from (1).

Corollary 4.3. We have isomorphisms of monoidal posets

$$
\begin{gathered}
\beta\left(P / p_{0}^{e_{0}} \cdots p_{n-1}^{e_{n-1}} P\right)=\prod_{i=0}^{n-1} \beta\left(\mathbb{Z} / 2^{e_{i}} \mathbb{Z}\right) \quad \text { and } \\
D L\left(\beta\left(P / p_{0}^{e_{0}} \cdots p_{n-1}^{e_{n-1}} P\right)\right)=\prod_{i=0}^{n-1} \mathbb{Z} / 2 .
\end{gathered}
$$

Corollary 4.4. For any PID $P$ and a non-zero element $q \in P$, the Bousfield lattice $\beta(P / q P)$ is monoidally distributive.

Proof. Noticing the relation

$$
\left(p_{0}^{s_{0}} \cdots p_{n-1}^{s_{n-1}}\right) \vee\left(p_{0}^{t_{0}} \cdots p_{n-1}^{t_{n-1}}\right)=p_{0}^{\ell_{0}} \cdots p_{n-1}^{\ell_{n-1}} \text { with } \ell_{i}=\min \left\{s_{i}, t_{i}\right\},
$$

the proof is straightforward.

Theorem 4.5. If $P$ is a PID and $q \in P \backslash\{0\}$, then $G R C 2$ holds on $\beta(P / q P)$, and so does GRC1. 
Proof. The ideal $N(\beta(P / q P))$ has the greatest element $g=p_{0} \cdots p_{n-1}$. We compute

$$
\begin{aligned}
\left(p_{0}^{s_{0}} \cdots p_{n-1}^{s_{n-1}}\right) \vee g & =p_{0}^{\min \left\{s_{0}, 1\right\}} \cdots p_{n-1}^{\min \left\{s_{n-1}, 1\right\}}=p_{0}^{\min \left\{2 s_{0}, 1\right\}} \cdots p_{n-1}^{\min \left\{2 s_{n-1}, 1\right\}} \\
& =\left(p_{0}^{2 s_{0}} \cdots p_{n-1}^{2 s_{n-1}}\right) \vee g=\left(p_{0}^{s_{0}} \cdots p_{n-1}^{s_{n-1}}\right)^{2} \vee g .
\end{aligned}
$$

So the theorem follows from Theorem 3.23.

Remark 4.6. We have another proof of the theorem. Since $\beta(P / q P)=\prod_{i=0}^{n-1} \beta\left(\mathbb{Z} / 2^{e_{i}} \mathbb{Z}\right)$ and GRC2 holds on $\beta\left(\mathbb{Z} / 2^{e_{i}} \mathbb{Z}\right)$, GRC2 holds on $\beta(P / q P)$ by Proposition 3.26.

\section{Bousfield lattices of Stable homotopy CATEGORIES}

Let $\mathcal{L}_{E}$ for a spectrum $E$ denote the stable homotopy category of $E$-local spectra, and $\mathbb{B}\left(\mathcal{L}_{E}\right)$ the Bousfield lattice in the sense of Bousfield. Then we have the Bousfield localization functor $L_{E}: \mathcal{S} \rightarrow \mathcal{L}_{E}$. The monoidal structure of $\mathcal{L}_{E}$ is given by $X Y=L_{E}(X \wedge Y)$. We consider the Johnson-Wilson spectra $E(n)$ and the Morava $K$-theories $K(n)$ for $n \geq 0$. By the chromatic viewpoint, investigating the categories $\mathcal{L}_{n}\left(=\mathcal{L}_{E(n)}\right)$ and $\mathcal{L}_{K(n)}$ is one of main targets of stable homotopy theory. We determine the Bousfield lattices of these categories.

We begin with a simple category. A spectrum $F$ is called a field if it is a ring spectrum and $F \wedge X=\bigvee \Sigma^{a} F$ for all spectra $X$.

Proposition 5.1. Let $F$ be a field. Then, $\mathbb{B}\left(\mathcal{L}_{F}\right)=\mathbb{Z} / 2$.

Proof. Since $F$ is a ring spectrum, we have $F X=F \wedge X$. We see easily $\langle X\rangle \geq\langle F X\rangle$. Suppose that $(F X) C=0$. Then, $X C$ is $F$-acyclic and so $X C=0$. It follows that $\langle X\rangle=\langle F X\rangle=\left\langle\bigvee \Sigma^{i} F\right\rangle=0$ or $\langle F\rangle$, which shows the lemma.

By [4], the Eilenberg-MacLane spectrum $H \mathbb{Z} / p$ and the Morava $K$-theories $K(n)$ are fields.

Corollary 5.2. $\mathbb{B}\left(\mathcal{L}_{H \mathbb{Z} / p}\right)=\mathbb{Z} / 2=\mathbb{B}\left(\mathcal{L}_{K(n)}\right)$.

Theorem 5.3. Let $p_{0}, \ldots, p_{n}$ be $n+1$ distinguished primes. Then $\mathbb{B}\left(\mathcal{L}_{n}\right)$ is isomorphic to $\beta\left(\mathbb{Z} / p_{0} \cdots p_{n}\right)=\prod_{i=0}^{n} \mathbb{Z} / 2$ in $\mathcal{M P}$.

Proof. The Bousfield lattice $\mathbb{B}\left(\mathcal{L}_{n}\right)$ consists of $\left\langle L_{n} X\right\rangle$ for all spectra $X$, which equals, by Ravenel [9],

$$
\begin{aligned}
&\left\langle L_{n} X\right\rangle=\left\langle L_{n} S^{0}\right\rangle \cdot\langle X\rangle=\langle E(n)\rangle \cdot\langle X\rangle \\
&=\left(\bigvee_{0 \leq i \leq n}\langle K(i)\rangle\right) \cdot\langle X\rangle=\bigvee_{0 \leq i \leq n} \text { and } K(i) \wedge X \neq 0 \\
&\langle K(i)\rangle .
\end{aligned}
$$

since $L_{n}$ is smashing and $K(n)$ is a field. Here $\langle X\rangle \cdot\langle Y\rangle$ is the Bousfield class of the smash product $X \wedge Y$. We define a map $f: \mathbb{B}\left(\mathcal{L}_{n}\right) \rightarrow \beta\left(\mathbb{Z} / p_{0} \cdots p_{n}\right)$ by $f\left(\bigvee_{i \in S}\langle K(i)\rangle\right)=\prod_{i \notin S} p_{i}$ for $S \subset\{0,1, \cdots, n\}$. Then $f$ preserves multiplication, since

$$
\begin{aligned}
\left(\bigvee_{i \in S}\langle K(i)\rangle\right)\left(\bigvee_{j \in T}\langle K(j)\rangle\right) & =\bigvee_{i \in S \cap T}\langle K(i)\rangle, \\
\left(\prod_{i \notin S} p_{i}\right)\left(\prod_{j \notin T} p_{j}\right) & =\prod_{i \notin S \text { or } i \notin T} p_{i}=\prod_{i \notin S \cap T} p_{i} .
\end{aligned}
$$

Moreover, for the order, we have

$$
\begin{aligned}
\bigvee_{i \in S}\langle K(i)\rangle \leq \bigvee_{i \in T}\langle K(i)\rangle & \Leftrightarrow S \subset T \Leftrightarrow I(n)-S \supset I(n)-T \\
& \Leftrightarrow \prod_{i \notin S} p_{i} \leq \prod_{i \notin T} p_{i},
\end{aligned}
$$

and $f$ is a monoidal poset map. 
A similar argument shows the following

Theorem 5.4. Let $E=\bigvee_{i \in F} K(i)$ be a spectrum for a finite subset $F$ of $\mathbb{Z}_{\geq 0}$. Then $\mathbb{B}\left(\mathcal{L}_{E}\right)$ is isomorphic to $\prod_{i \in F} \mathbb{Z} / 2$.

This together with Theorem 4.5 implies

Corollary 5.5. GRC2 holds on $\mathbb{B}\left(\mathcal{L}_{E}\right)$ for a spectrum $E=\bigvee_{i \in F} K(i)$ on a finite subset $F$ of $\mathbb{Z}_{\geq 0}$.

The chromatic tower $\mathcal{L}_{0} \leftarrow \mathcal{L}_{1} \leftarrow \mathcal{L}_{2} \leftarrow \cdots$ induces the inverse system

$$
\mathbb{B}\left(\mathcal{L}_{0}\right) \leftarrow \mathbb{B}\left(\mathcal{L}_{1}\right) \leftarrow \mathbb{B}\left(\mathcal{L}_{2}\right) \leftarrow \cdots
$$

Moreover, we notice that $B_{\infty}:=\lim _{n} \mathbb{B}\left(\mathcal{L}_{n}\right)=\prod_{n} \mathbb{Z} / 2$ in $\mathcal{M P}$. We call a spectrum harmonic if it is $\left(\bigvee_{i \geq 0} K(i)\right)$-local.

Theorem 5.7. Let $\mathcal{H}$ be the stable homotopy category of harmonic spectra. Then $\mathbb{B}(\mathcal{H})$ is isomorphic to $B_{\infty}$ in $\mathcal{M P}$.

Proof. Let $f: \prod \mathbb{Z} / 2 \rightarrow \mathbb{B}(\mathcal{H})$ be the poset map defined by $\left(x_{n}\right) \mapsto \bigvee_{x_{n}=1}\langle K(n)\rangle$ and let $p_{n}: \mathbb{B}(\mathcal{H}) \rightarrow \mathbb{B}\left(\mathcal{L}_{n}\right)$ be the poset map defined by $\langle X\rangle \mapsto\langle X\rangle \cdot\langle E(n)\rangle$. Then, we have the following commutative diagram

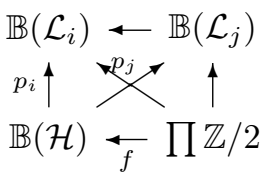

for any $i$ and $j$ with $i \leq j$, since

$$
\begin{aligned}
p_{i} f\left(\left(x_{n}\right)\right) & =p_{i}\left(\bigvee_{x_{n}=1}\langle K(n)\rangle\right)=\bigvee_{x_{n}=1}\langle K(n)\rangle \cdot\langle E(i)\rangle \\
& =\bigvee_{i \geq n, x_{n}=1}\langle K(n)\rangle .
\end{aligned}
$$

Therefore, $\mathbb{B}(\mathcal{H})$ is the inverse limit of the above system (5.6) by definition.

Proof of Theorem 1.2. This follows from Theorem 5.7 and Proposition 3.26.

In the same way, we obtain

Theorem 5.8. Let $T$ be a set of field spectra, and put $\bigvee T=\bigvee_{F \in T} F$. Then, $\mathbb{B}(\mathcal{L} \vee T)=\prod \mathbb{Z} / 2$.

\section{Problems}

We leave some problems in this section.

Problem 6.1. What is a condition on $X \stackrel{f}{\rightarrow} Y$ in $\mathcal{M}_{0}^{e p i}$, under which $\beta(f)$ is an isomorphism?

Suppose that the problem is settled and we find a map from $\mathbb{B}$ to a commutative monoid $Y$ such that $\beta(f)$ is an isomorphism. Then, we may study $\mathbb{B}=\beta(\mathbb{B})$ by observing $\beta(Y)$ by virtue of Proposition 2.16, which may let us consider the lattice from a different viewpoint.

Problem 6.2. Let $M$ be a monoid with 0 . Then, is there a ring $R$ such that $\beta(M)$ is isomorphic to $R$ as a monoid? 
Example 6.3. Let $p_{0}, \ldots, p_{n}$ be $n+1$ distinguished primes. Then $\beta\left(\mathbb{Z} / p_{0} \ldots p_{n}\right)=$ $\prod_{i=0}^{n} \mathbb{Z} / 2$ as a monoid by Theorem 5.3.

If this is possible, we may approach these from the ring theoretic viewpoint.

Problem 6.4. Are $\mathbb{B} / J(h)$ and $\mathbb{D L}$ monoidal posets?

\section{REFERENCES}

1. A. K. Bousfield, The Boolean algebra of spectra, Comment. Math. Helv. 54 (1979), 368-377.

2. W. G. Dwyer and J. H. Palmieri, Ohkawa's theorem: there is a set of Bousfield classes. Proc. Amer. Math. Soc., 129 (2001), 881-886.

3. W. G. Dwyer and J. H. Palmieri, The bousfield lattice for truncated polynomial algebras, Homology, Homotopy and Applications, 10 (2008), 413-436.

4. M. J. Hopkins and J. H. Smith, Nilpotence and stable homotopy theory. II, Ann. Math. 148 (1998), 1-49.

5. M. Hovey and J. H. Palmieri, The structure of the Bousfield lattice, Contemp. Math. 239 (1999), 175-196.

6. M. Hovey, J. H. Palmieri and N. P. Strickland, Axiomatic stable homotopy theory, Mem. Am. Math. Soc. 610 (1997).

7. S. B. Iyengar and H. Krause, The Bousfield lattice of a triangulated category and stratification, Math. Z. 273 (2013), 1215-1241.

8. T. Ohkawa, The injective hull of homotopy types with respect to generalized homology functors, Hiroshima Math. J. 19 (1989), 631-639.

9. D. C. Ravenel, Localization with respect to certain periodic homology theories, Amer. J. Math. , 106 (1984), 351-414.

Graduate school of Mathematics, Nagoya University, Furo-cho, Chikusa-Ku, Nagoya 464-8602, JAPAN

E-mail address: ryo_kato_1128@yahoo.co.jp

Department of Mathematics, Faculty of Science, Kochi University, Kochi, 780-8520, JAPAN

E-mail address: katsumi@kochi-u.ac.jp

Department of Mathematics, Faculty of Science, Kochi University, Kochi, 780-8520, JAPAN

E-mail address: y.tatehara@gmail.com 